\title{
Intratumoral LAG-3 + cells infiltration determines poor prognosis in patients with castration-naiive prostate cancer
}

Bo Dai ( $\sim$ bodai1978@126.com )

Fudan University Shanghai Cancer Center

Nan Lin

Fudan University Shanghai Cancer Center

Jun-hong Li

Fudan University Shanghai Cancer Center

Yun-jie Yang

Fudan University Shanghai Cancer Center

Jun-Yu Zhang

Fudan University Shanghai Cancer Center

Xuan-zhi Zhang

Fudan University Shanghai Cancer Center

Zheng Liu

Fudan University Shanghai Cancer Center

\section{Research Article}

Keywords:

Posted Date: February 24th, 2022

DOI: https://doi.org/10.21203/rs.3.rs-1383643/v1

License: (9) This work is licensed under a Creative Commons Attribution 4.0 International License. Read Full License 
TITLE: Intratumoral LAG-3+ ${ }^{+}$cells infiltration determines poor prognosis in patients with castration-naïve prostate cancer.

Nan Lin ${ }^{1, \dagger}$, Jun-hong Lij ${ }^{1} \dagger$, Yun-jie Yang ${ }^{1, \dagger}$, Jun-Yu Zhang ${ }^{1}$, Xuan-zhi Zhang ${ }^{1}$, Zheng Liu ${ }^{1}$, Bo Dai ${ }^{1, *}$.

1. Department of Urology, Fudan University Shanghai Cancer Center, Shanghai 200032, China.

$\dagger$ These authors have contributed equally to this work.

\section{${ }^{*}$ Corresponding authors:}

Bo Dai, MD, Department of Urology, Fudan University Shanghai Cancer Center, 270 Dongan Road, Xuhui District, Shanghai 20032, China (bodai1978@126.com);

\section{Abstract}

Background: As a transmembrane immune checkpoint protein, lymphocyteactivation gene 3 (LAG-3) is expressed on activated T cells and natural killer cells, could negatively regulate $\mathrm{CD} 8^{+} \mathrm{T}$ cells activation and reshaped the phenotype of $\mathrm{CD} 4^{+} \mathrm{T}$ cells to trigger an immunosuppressive function. Previous studies had demonstrated the prognostic value of LAG-3 in different cancers. However, its value in evaluating prognosis and predicting ADT resistance in patients with prostate cancer is still unclear.

Method and Materials: The mRNA sequencing data of 498 and 140 prostate cancer specimens were respectively from TCGA and MSKCC. 175 patients with castration-naïve prostate cancer between June 2012 and December 2014 from Fudan University Shanghai Cancer Center at first visit were retrospective analyzed to evaluate the prognostic value of $\mathrm{LAG}_{-} 3^{+}$cells through immunohistochemistry. All patients were treated with androgen deprivation 
therapy after initial diagnosis. Fresh tumor tissue samples from 18 patients with prostate cancer were examined to discover the phenotype of the immune cells by flow cytometry.

Results: High infiltration of intratumoral LAG- $3^{+}$cells predicted poor castrationresistant prostate cancer-free survival (CRPC-FS, $P=0.0006)$ and overall survival (OS, $P<0.0001)$ in castration-naïve prostate cancer. Multivariate Cox regression model indicated that high infiltration of intratumoral LAG- $3^{+}$cells was an independent risk factor for shorter CRPC-FS (hazard ratio [HR], 1.90; 95\%confidence interval $[\mathrm{Cl}], 1.30-2.77 ; \mathrm{P}=0.001$ ) as well as shorter OS (hazard ratio [HR], 2.82; 95\%confidence interval [Cl],1.65-4.80; $\mathrm{P}<0.001$ ). Intratumoral LAG- $3^{+}$cell abundance correlated with exhausted $\mathrm{CD} 8^{+} \mathrm{T}$-cells in castration-naïve prostate cancer, marking dampened $\mathrm{CD}^{+} \mathrm{T}$-cell antitumor immunity.

Conclusion: This study demonstrated that intratumoral LAG- $3^{+}$cell abundance in patients with castration-naïve prostate cancer was associated with earlier resistance to ADT and shorter overall survival. Intratumoral $L A G-3^{+}$cell abundance impairs $\mathrm{CD} 8^{+} \mathrm{T}$-cell antitumor immunity in patients with castrationnaïve prostate cancer.

\section{Introduction}

Prostate cancer ( $\mathrm{PCa}$ ) is the second most common cancer in men worldwide, with an increasing incidence rate. Although PSA screening helps detect earlystage prostate cancer, there were over 300,000 patients die from it ever year ${ }^{[1]}$.Since the emergence of new therapies for late-stage prostate cancer, overall survival has been prolonged, however a large proportion of patients, such as metastatic castration-naïve prostate cancer (mCNPC) patients, still have a poor 5 -year survival ${ }^{[2,3]}$. 
deprivation therapy (ADT) ${ }^{[4]}$. Unfortunately, nearly all patients with CNPC would eventually develop to castration-resistant status, named castration resistant prostate cancer (CRPC). Considering that we don't currently have an effective treatment for CRPC, which means that it is necessary to provide the most appropriate therapy for the patients with CNPC.

Biomarkers for risk stratification and therapy response are still lacking in $\mathrm{CNPC}^{[5]}$. Previous studies had demonstrated the prognostic value of lymphocyte-activation gene 3 (LAG-3), an immune checkpoint receptor protein, in different cancers. In breast cancer, LAG-3 ${ }^{+}$cells abundance indicated improved prognosis and might be the counterbalance reaction to the ongoing antitumor T cell immunity ${ }^{[6]}$. Inversely, LAG-3+ cell infiltration was associated with poor survival in non-small cell lung cancer, representing an immunosuppressive landscape ${ }^{[7]}$. As a transmembrane protein discovered in 1990, LAG-3 is expressed on activated T cells and natural killer cells ${ }^{[8,9]}$. Several preclinic studies have confirmed that LAG-3 signaling led to cytotoxic CD8+ $T$ cells exhaustion, and LAG-3 blockade can synergize with PD-1/PDL1 therapy to reinvigorate antitumor immunity ${ }^{[10,11] .}$

Herein, our purpose was to elucidate the prognostic significance of LAG-3 ${ }^{+}$ cells and its relationship with ADT benefit in prostate cancer. For these reasons, we conduct this study to evaluate the expression status and prognostic value of LAG $-3^{+}$cells in prostate cancer. Meanwhile, the association of LAG-3 mRNA expression with prognosis and immune composition was studied based on The Cancer Genome Atlas (TCGA) and Memorial Sloan Kettering Cancer Center (MKSCC). Furthermore, we discovered the correlation between LAG $-3^{+}$cell abundance and the phenotype of the $\mathrm{CD} 8^{+} \mathrm{T}$ cells by flow cytometry. This work is the first exploration of the comprehensive clinical value of $\mathrm{LAG}-3^{+}$cells in CNPC. 


\section{Materials and method}

\section{Study cohort}

Prostate cancer tissue specimens were obtained from 205 patients diagnosed as de novo CNPC who received prostate biopsy or Trans-Urethral Resection of the Prostate (TURP) between June 2012 and December 2014 at the Fudan University Shanghai Cancer Center (FUSCC). Diagnosis of prostate cancer were based upon transrectal ultrasound-guided 12 to 18 core biopsies of the prostate. Metastatic sites were defined by the results of Emission Computed Tomography (ECT), pelvic magnetic resonance imaging (MRI), thoracic and abdominal Computed Tomography (CT) scan. Additional MRI were asked for patients with unequivocal skeletal metastatic sites to assure the diagnosis of bone lesions. High metastatic volume prostate cancer was defined as existence of visceral metastasis or more than three bone lesions with at least one except the vertebral column or pelvis ${ }^{[12]}$. All patients received traditional ADT using $3.6 \mathrm{mg}$ goserelin, administered subcutaneously each month, plus bicalutamide, $50 \mathrm{mg}$ orally each day until the development of CRPC. CRPC was defined as documented biochemical progression (three consecutive increases in PSA concentrations 1 week apart, resulting in two 50\% increases over the nadir, and PSA $>2 \mathrm{ng} / \mathrm{mL}$ ) or radiological progression (the appearance of new lesions: 1 or $\geq 2$ bone lesions on bone scan, or a soft tissue lesion as defined by the Response Evaluation Criteria in Solid Tumours) and testosterone $<50 \mathrm{ng} / \mathrm{dL}$ or $1.7 \mathrm{nmol} / \mathrm{L}^{[13]}$. Because of the immunohistochemistry (IHC) detachment, a few specimens were lost on the TMA. Therefore, 175 eligible patients with prostate cancer were included. At the end of follow-up time, 116 patients progressed to CRPC. Among these CRPC patients, 36 patients (42.86\%) used abiraterone as first line therapy and 27 patients (32.14\%) used the docetaxel. Formalin-fixed paraffinembedded samples of FUSCC were sectioned by tissue microarray (TMA) 
technology. The clinical-pathological characteristics of 175 patients with prostate cancer are presented in Table 1.

The following characteristics were acquired from medical records: age, hemoglobin (HGB), albumin (ALB), lactate dehydrogenase (LDH), alkaline phosphatase (ALP), prostate specific antigen (PSA), Gleason score, Eastern Cooperative Oncology Group (ECOG) score, treatment regimens, and clinical outcomes.

\section{Immunohistochemistry}

IHC staining was performed on formalin-fixed, paraffin-embedded tissue microarray (TMA) as described previously [24]. The IHC antibodies are listed in Supplementary Table 1. In brief, the slides were baked at $60^{\circ} \mathrm{C}$ for 6 hours, deparaffinized in xylene (three times, 15 min each) and rehydrated in graded alcohol. Next, the slides were immersed in sodium citrate buffer $(0.01 \mathrm{M}$ sodium citrate buffer, $\mathrm{pH}=6$ ) for antigen retrieval and then blocked with $3 \% \mathrm{H}_{2} \mathrm{O}_{2}$ in methanol at $37^{\circ} \mathrm{C}$ for $30 \mathrm{~min}$. The slides were incubated with the primary antibodies at $4^{\circ} \mathrm{C}$ overnight and visualized by $3,3^{\prime}$-diaminobenzidine (DAB) stain system. All TMA slides were evaluated under Leica DM6000 B Microsystems by two sophisticated pathologists independently, who were blinded to clinical data. The positive cells were enumerated from the representative view of the three sections in high-power field (HPF, $\times 200$ magnification), and the mean value was adopted. The cut-off of low/high LAG$3^{+}$cells subgroups was determined by median value ( 6 cells/HPF).

\section{Differential expression analysis}

The mRNA sequencing data of 498 prostate cancer specimens in TCGA and the data of 140 prostate cancer specimens in MSKCC were both downloaded 
from http://www.cbioportal.org/ in October 2020. The RNA-seq data were obtained as Fragments Per Kilobase of transcript per Million mapped reads (FPKM), and the mRNA expression of 498 patients with prostate cancer from TCGA were normalized by the formula $\log 2(\mathrm{FPKM}+1)$ before analyses. Because of 6 specimens lacked part of survival data, 490 prostate cancer samples were included in survival analyses and 498 samples were involved in further GSEA analyses or immune cells infiltration distributions analyses. Similarly, 140 prostate cancer specimens were available for prognostic analysis in MSKCC. The cut-off value of LAG-3 mRNA expression was determined by median value.

Gene set enrichment analyses (GSEA) analyses were applied to determine the biological pathway divergences between high and low LAG-3 mRNA expression in TCGA cohort. The CIBERSORT method ${ }^{[14]}$, an algorithm to evaluate hematopoietic cell distribution from tumor RNA mixtures, was used to analyze the differential immune cells infiltration distributions.

\section{Flow cytometry}

Fresh samples of prostate cancer, including tumor tissues $(n=18)$, were collected from Fudan University Shanghai Cancer Center. Single-cell suspension was performed as described ${ }^{[15]}$. Then samples were stained with the indicated $\mathrm{mAbs}$ for $30 \mathrm{~min}$ at $4^{\circ} \mathrm{C}$ after lysing red blood cells. Cells were stimulated for 5 hours with phorbol myristate acetate $(50 \mathrm{ng} / \mathrm{mL})$ and ionomycin $(1 \mu \mathrm{g} / \mathrm{mL})$ in the presence of GolgiStop protein transport inhibitor (1:1000) for intracellular cytokine measurement. Cells were stained with interested surface markers, and Fixation/Permeabilization Solution Kit (BD Biosciences) was used for intracellular protein staining according to the manufacturer's instructions. Stained cells were washed and resuspended in phosphate-buffered saline/0.1\% bovine serum albumin coupled with azide. Flow cytometry data were analyzed 
by FlowJo software (Tree Star, San Carlos, California, USA). All flow cytometry antibodies are listed in Supplementary Table 2.

\section{Statistical analysis}

Patients' characteristics were summarized using descriptive methods. Continuous variables were presented by median and range. Categorical variables were summarized by number and frequency. Parametric ( $t$ test) and nonparametric (Chi-square test or Mann-Whitney $U$ test) tests were applied for comparisons between two groups. Progression-free survival (PFS), overall survival (OS) and castration-resistant prostate cancer-free survival (CRPC-FS) were estimated using Kaplan-Meier survival analysis. PFS was defined as the time from commencing ADT to the appearance of disease-related events. CRPC-FS was defined as the time from commencing ADT to the date of progression to CRPC. Univariate and multivariate Cox analysis were conducted to evaluate each factor's impact on survival. A two-tailed $p$ value of $<0.05$ was considered statistically significant in our study. All statistical analyses were conducted using IBM SPSS Statistics V.25.0, R V.3.5.1 and GraphPad Prism Software V.9.0.

\section{Results}

\subsection{Associations between $\mathrm{LAG}^{+}{ }^{+}$cells infiltration and patients' clinicopathologic features}

Of the 175 patients included in this study, the median age was 68 . Median PSA at diagnosis was 154 , ranging from $10 \mathrm{ng} / \mathrm{ml}$ to $5000 \mathrm{ng} / \mathrm{ml}$. The biopsy results showed that $66.29 \%$ of these patients presented Gleason score $\geq 9$ disease. The majority of these patients $(89.71 \%)$ were of $\mathrm{M} 1 \mathrm{~b}$ stage, while visceral metastases were observed in 4 patents. 72 and 103 patients were classified 
into low metastatic volume and high metastatic volume separately according to the CHAARTED standard ${ }^{[12]}$. After a median follow-up of 39 months, 116 patients developed CRPC and 66 died at the end of this study. The average time to mCRPC was 18.23 months. When diseases progressed to mCRPC, 27 (32.14\%) patients adopted docetaxel as first line therapy and 36 (42.86\%) adopted abiraterone (Table 1).

Representative immunohistochemistry images of LAG-3-positive-staining were illustrated in Figure 1A. LAG-3+ cells were stained clearly within tissues, and the infiltration differed distinctly in different patients. We then separately counted the positive cells from the representative view of the three sections in HPF (400x). The cut-off of low/high LAG- $3^{+}$cells subgroups was determined by median value ( 6 cells/HPF).

In the FUSCC cohort, $84(48.00 \%)$ had high level of LAG-3+ cell infiltration, there was no differences in the total Gleason score between two patients with high versus low $L A G-3^{+}$cell infiltration ( $p=0.063$, by Chi-square test). The two groups also did not have statistically differences in the following clinical characteristics: age, perineural invasion (PNI), ECOG performance status, metastatic volume and so on (Table 2).

\subsection{Intratumoral LAG $-3^{+}$cells enrichment indicates poor clinical outcomes in patients with castration-naïve prostate cancer}

The TCGA cohort and the MSKCC cohort were divided into two subgroups by the median expression of LAG-3 mRNA respectively. Kaplan-Meier survival curves showed that prostate cancer patients with high LAG-3 mRNA expression had significantly shorter progression-free survival (PFS, $p=0.0009$ ) in TCGA cohort and shorter progression-free survival in MSKCC cohort (PFS, $p=0.0485$ ) (Figure 1B). 
Kaplan-Meier survival curves of the FUSCC cohort showed that patients with high intratumoral LAG-3 ${ }^{+}$cell infiltration had significantly shorter castrationresistant prostate cancer-free survival (CRPC-FS, $P=0.006$ ) and overall survival (OS, $\mathrm{P}<0.0001$ ) than those with low intratumoral LAG $-3^{+}$cell infiltration (Figure 1C).

Univariate analysis showed that reduced ALB, ECOG PS $\geq 2$, high metastatic volume and presence of perineural invasion (PNI) were significantly associated with shorter OS, as well as high $\mathrm{LAG3}^{+}$cell infiltration. When these factors were included in a multivariate cox model, ALB and $\mathrm{LAG}^{+}$cell infiltration were independent risk factors of CRPC-FS. The adjusted HR was 2.82(1.65 to 4.80, $\mathrm{P}<0.001$ ) for $\mathrm{LAG}^{+}$cell infiltration in predicting CRPC-FS. Factors associated with CRPC-FS in univariate analysis included ALB, LDH, ALP, pretreatment PSA, ECOG PS, metastatic volume, perineural invasion and $\mathrm{LAG}^{+}$cell infiltration. Multivariate analysis showed that $\mathrm{LAG}^{+}$cell infiltration was also independent prognostic factors in predicting CRPC-FS. The adjusted HR was 1.90 (1.30 to 2.77, $\mathrm{P}=0.001$ ) for $\mathrm{LAG3}^{+}$cell infiltration in predicting CRPC-FS (Table 3).

\subsection{Intratumoral LAG $-3^{+}$cell abundance impairs CD8+ T cell antitumor immunity in patients with prostate cancer}

In order to explore the cellular explanation for favorable prognostic value of low $\mathrm{LAG}^{+}$cell infiltration. We first processed data from TCGA by bioinformatics methods. The results of Gene Set Enrichment Analysis (GSEA) were illustrated in Figure 2A, multiple T lymphocyte-related signal pathways were enriched in high LAG-3 mRNA expression group. Additionally, CIBERSORT analysis showed that high LAG-3 mRNA expression group had higher infiltration of CD8 ${ }^{+}$ T cells, Tregs, resting masT cells than those with low LAG-3 mRNA expression. 
These results suggested that high $\mathrm{CD} 8^{+} \mathrm{T}$ cells infiltration might correlate with high LAG-3 mRNA expression (Figure 2B-C). Interestingly, previous research found that high intratumoral $\mathrm{CD}^{+} \mathrm{T}$ cell infiltration is associated with improved survival in prostate cancer patients ${ }^{[16]}$.

Based on the 'paradoxical' discovery from prostate cancer patients in TCGA, we sought to clarify on the relationship between LAG-3 ${ }^{+}$infiltration and intratumoral $\mathrm{CD}^{+} \mathrm{T}$ cells in prostate cancer by flow cytometry. The global characterization of $\mathrm{CD}^{+} \mathrm{T}$ cells was subsequently investigated according to LAG- $3^{+}$cell abundance. We found that the proliferative ability (Ki-67) and actual infiltration of $\mathrm{CD}^{+} \mathrm{T}$ cell were increased in high $\mathrm{LAG}-3^{+}$cells infiltration tumors (Figure 3A-B). Additionally, $\mathrm{CD} 8^{+} \mathrm{T}$ cells in $\mathrm{LAG}-3^{+}$cells high infiltration tumors expressed increased immune checkpoints, including PD-1, CTLA-4, TIGIT and TIM-3, while expressing decreased effector cytokines (IFN-y) (Figure 3C-D). The relationship between $\mathrm{LAG}-3^{+} \mathrm{CD} 8^{+} \mathrm{T}$ cells proportion and other immune checkpoint markers of $\mathrm{CD}^{+}{ }^{+}$T cells (PD-1, TIGIT, CTLA-4 and TIM-3) were shown in Supplementary Figure 1. These results indicated that high LAG-3 ${ }^{+}$ cells infiltration was associated with exhausted $\mathrm{CD} 8^{+} \mathrm{T}$ cells infiltration, which led to poor outcomes in patients with prostate cancer.

\section{Discussion}

Although we define ADT as the gold-standard therapy for CNPC patients, not all of them could benefit from it. Appropriate risk classification of patients with CNPC can help identify which patient can benefit more from ADT. As a conventional staging system, pathological stratification did not pay much attention to the immune reaction between tumor and the host. Expanding researches pointed that tumor immune-profiling and immune contexture inevitably touched the prognosis and therapeutic efficacy of patients ${ }^{[17,18]}$, and raised novel opinions that tumor immune environment may guide clinical 
decision making and select most suitable therapy for each patient. In the initially study, we explored the impact of LAG-3, which is a novel immune checkpoint molecule, on prognosis and tumor microenvironment by the data of prostate cancer specimens in TCGA and MSKCC. Further research evaluated 175 prostate cancer patients from FUSCC. Our results suggested that high infiltration of intratumoral LAG $-3^{+}$cells might be an independent unfavorable prognosticator for both OS and CRPC-FS of prostate cancer patients. Current findings revealed that LAG-3 expressed on $\mathrm{CD}^{+} \mathrm{T}$ cells might negatively regulated T cells cytotoxicity and activation, and LAG-3 expressed on $\mathrm{CD} 4^{+} \mathrm{T}$ cells reshaped its phenotype to trigger an immunosuppressive function ${ }^{[10]}$, supporting our observation that high LAG- $3^{+}$cells infiltration marked exhausted $\mathrm{CD}^{+} \mathrm{T}$ cells in castration-naïve prostate cancer. Eventually lead to the poor clinical outcomes in LAG-3 ${ }^{+}$cells-high subgroup, reflecting the interaction between cancer and immune system.

Presently, $\mathrm{CD}^{+} \mathrm{T}$ cells were regarded as a protective factor in various solid tumors, including prostate cancer ${ }^{[16,19]}$. However, $\mathrm{CD}^{+} \mathrm{T}$ cells remain heterogeneous, and several subtypes of intratumoral $\mathrm{CD}^{+} \mathrm{T}$ cells are associated with poor clinical outcomes ${ }^{[20]}$. LAG-3, a novel coinhibitory receptor, can promote tumor growth and drive the exhaustion of tumor-infiltrating lymphocytes, including $\mathrm{CD} 8^{+} \mathrm{T}$ cells in multiple cancer types. In this study, we reported that intratumoral LAG $-3^{+}$cell abundance was positively correlated with ADT resistance, poor prognosis and $\mathrm{CD}^{+} \mathrm{T}$ cells exhausted, marking dampened $\mathrm{CD} 8^{+} \mathrm{T}$-cell antitumor immunity, through the retrospective analysis of a considerable population. In other words, CNPC patients with lower intratumoral LAG $-3^{+}$cell infiltration might more possibly to benefit from standard androgen deprivation therapy.

Recently, immune checkpoint block (ICB) therapy showed a confirmed promising efficacy in multiple tumor type, by restoring and boosting antitumor 
immune response to conquer tumor escape. However, only a small proportion of patients with advanced prostate cancer obtained durable remission from ICB therapy in clinical trials (Pembrolizumab, ORR: 17.4\%) ${ }^{[21]}$, uncovering that a future emphasis on ICB therapy is to find combination-based strategies to optimize effectiveness. Given its significant role in T cells exhaustion, LAG-3 has been evaluated as a potential ICB target in malignancies and autoimmune diseases ${ }^{[10]}$. Our preclinical data based on samples of patients with prostate cancer demonstrated LAG- $3^{+}$cells infiltration was strongly correlated with $\mathrm{CD} 8^{+}$ $T$ cells dysfunction and ICK expression (PD-1/TIGIT), supporting further evaluation of LAG-3 targeted agent for single or combined ICB therapy in clinical trials of CNPC.

In conclusion, this study based on standardly treated de-novo CNPC cohort demonstrated that LAG-3 $3^{+}$cells abundance was an independent predictor for inferior prognosis and ADT resistance in castration-naïve prostate cancer, simultaneously discovered the correlation between $\mathrm{LAG}-3^{+}$cells and $\mathrm{CD} 8^{+} \mathrm{T}$ cells dysfunction. Molecular features of LAG-3-high tumors implied guiding significance of LAG-3 expression in personalized approaches to therapy. We recommended that future researches could focus on LAG- $3^{+}$cells infiltration as an important biomarker in the prognosed or therapeutic landscape of castrationnaïve prostate cancer.

\section{Reference}

[1] BRAY F, FERLAY J, SOERJOMATARAM I, et al. Global cancer statistics 2018: GLOBOCAN estimates of incidence and mortality worldwide for 36 cancers in 185 countries [J]. CA: a cancer journal for clinicians, 2018, 68(6): 394-424.

[2] WU J N, FISH K M, EVANS C P, et al. No improvement noted in overall or cause-specific survival for men presenting with metastatic prostate cancer over a 20 -year period []]. Cancer, 2014, 120(6): 818-23.

[3] STEELE C B, LI J, HUANG B, et al. Prostate cancer survival in the United States by race and stage (2001-2009): Findings from the CONCORD-2 study []]. Cancer, 2017, 123 Suppl 24(Suppl 24): $5160-77$. 
[4] BELDERBOS B P S, DE WIT R, LOLKEMA M P J, et al. Novel treatment options in the management of metastatic castration-naïve prostate cancer; which treatment modality to choose? [J]. Ann Oncol, 2019, 30(10): 1591-600.

[5] SEKINO Y, HAN X, BABASAKI T, et al. Microtubule-associated protein tau (MAPT) promotes bicalutamide resistance and is associated with survival in prostate cancer []]. Urologic oncology, 2020, 38(10): 795.e1-.e8.

[6] BURUGU S, GAO D, LEUNG S, et al. LAG-3+ tumor infiltrating lymphocytes in breast cancer: clinical correlates and association with PD-1/PD-L1+ tumors []]. Ann Oncol, 2017, 28(12): 2977 84.

[7] HE Y, YU H, ROZEBOOM L, et al. LAG-3 Protein Expression in Non-Small Cell Lung Cancer and Its Relationship with PD-1/PD-L1 and Tumor-Infiltrating Lymphocytes []]. Journal of thoracic oncology : official publication of the International Association for the Study of Lung Cancer, 2017, 12(5): 814-23.

[8] ANDERSON A C, JOLLER N, KUCHROO V K. Lag-3, Tim-3, and TIGIT: Co-inhibitory Receptors with Specialized Functions in Immune Regulation [J]. Immunity, 2016, 44(5): 989-1004. [9] TRIEBEL F, JITSUKAWA S, BAIXERAS E, et al. LAG-3, a novel lymphocyte activation gene closely related to CD4 [J]. The Journal of experimental medicine, 1990, 171(5): 1393-405. [10] ANDREWS L P, MARCISCANO A E, DRAKE C G, et al. LAG3 (CD223) as a cancer immunotherapy target []]. Immunological reviews, 2017, 276(1): 80-96.

[11] LICHTENEGGER F S, ROTHE M, SCHNORFEIL F M, et al. Targeting LAG -3 and PD-1 to Enhance T Cell Activation by Antigen-Presenting Cells [J]. Frontiers in immunology, 2018, 9(385. [12] SWEENEY C J, CHEN Y H, CARDUCCI M, et al. Chemohormonal Therapy in Metastatic Hormone-Sensitive Prostate Cancer []]. N Engl J Med, 2015, 373(8): 737-46.

[13] CORNFORD P, BELLMUNT J, BOLLA M, et al. EAU-ESTRO-SIOG Guidelines on Prostate Cancer. Part II: Treatment of Relapsing, Metastatic, and Castration-Resistant Prostate Cancer []]. Eur Urol, 2017, 71(4): 630-42.

[14] NEWMAN A M, LIU C L, GREEN M R, et al. Robust enumeration of cell subsets from tissue expression profiles []]. Nature methods, 2015, 12(5): 453-7.

[15] LIN C, HE H, LIU H, et al. Tumour-associated macrophages-derived CXCL8 determines immune evasion through autonomous PD-L1 expression in gastric cancer [J]. Gut, 2019, 68(10): 1764-73.

[16] YANG Y, ATTWOOD K, BSHARA W, et al. High intratumoral CD8(+) T-cell infiltration is associated with improved survival in prostate cancer patients undergoing radical prostatectomy []]. The Prostate, 2021, 81(1): 20-8.

[17] COFFELT S B, DE VISSER K E. Immune-mediated mechanisms influencing the efficacy of anticancer therapies []]. Trends in immunology, 2015, 36(4): 198-216.

[18] FRIDMAN W H, ZITVOGEL L, SAUTèS-FRIDMAN C, et al. The immune contexture in cancer prognosis and treatment []]. Nature reviews Clinical oncology, 2017, 14(12): 717-34.

[19] MATSUMOTO H, THIKE A A, LI H, et al. Increased CD4 and CD8-positive T cell infiltrate signifies good prognosis in a subset of triple-negative breast cancer [J]. Breast cancer research and treatment, 2016, 156(2): 237-47.

[20] THOMPSON E D, ZAHURAK M, MURPHY A, et al. Patterns of PD-L1 expression and CD8 T cell infiltration in gastric adenocarcinomas and associated immune stroma []]. Gut, 2017, 66(5): 794-801. 
[21] HANSEN A R, MASSARD C, OTT P A, et al. Pembrolizumab for advanced prostate

adenocarcinoma: findings of the KEYNOTE-028 study []]. Ann Oncol, 2018, 29(8): 1807-13.

\section{Statements \& Declarations}

\section{Funding}

This work was supported by a grant from the Medical Innovation Research Project of the Science and Technology Commission of Shanghai Municipality (20Y11905000); a grant from the Natural Science Foundation of Science and Technology Commission of Shanghai Municipality (20ZR1412300); and two grants from the AoXiang Project of the Shanghai anti-cancer association (SACA-AX201908, SACA-AX202005).

\section{Competing Interests}

The authors have no relevant financial or non-financial interests to disclose

\section{Author Contributions}

All authors contributed to the study conception and design. Material preparation, data collection and analysis were performed by Nan Lin, Junhong Li and Yunjie Yang. The first draft of the manuscript was written by Nan Lin and all authors commented on previous versions of the manuscript. All authors read and approved the final manuscript

\section{Data Availability}

The public database datasets generated during and/or analysed during the current study are available in the TCGA or MSKCC repository, http://www.cbioportal.org/.

Other datasets generated during and/or analysed during the current study are available from the corresponding author on reasonable request.

\section{Ethics approval}

This study was performed in line with the principles of the Declaration of Helsinki. Approval was granted by the Ethics Committee of Fudan University. Approval forms are available from the corresponding author on reasonable request.

\section{Consent to participate}

Informed consent was obtained from all individual participants included in the study.

\section{Consent to publish}

The authors affirm that human research participants provided informed consent for publication of the images in all Figures 
A

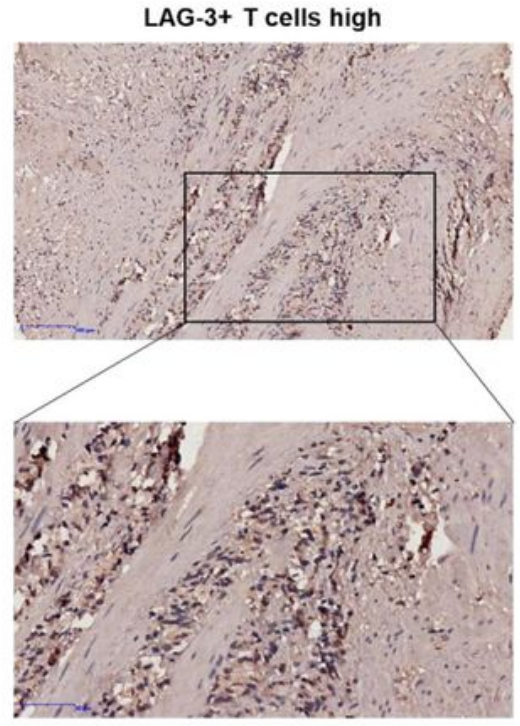

B

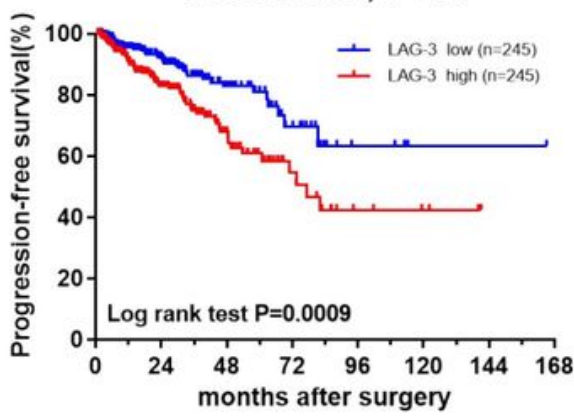

C

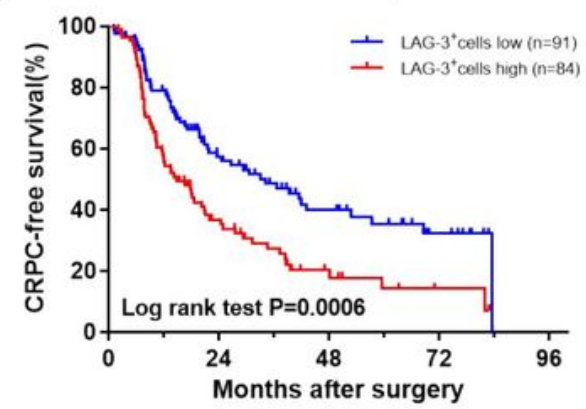

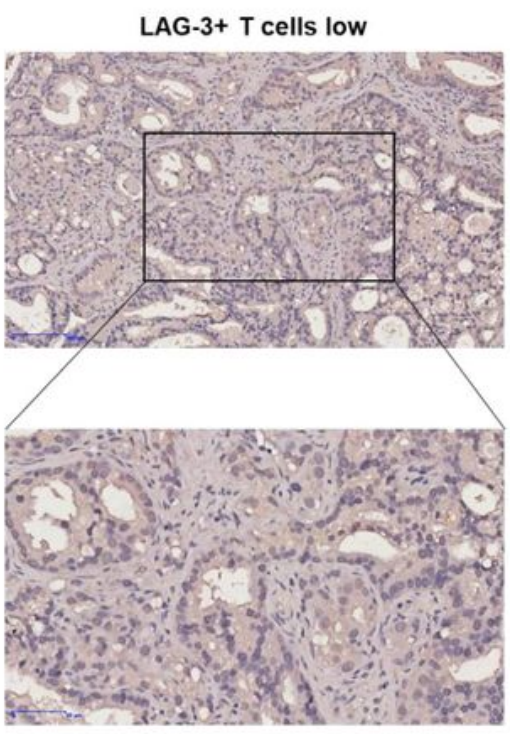

MSKCC cohort, $n=140$

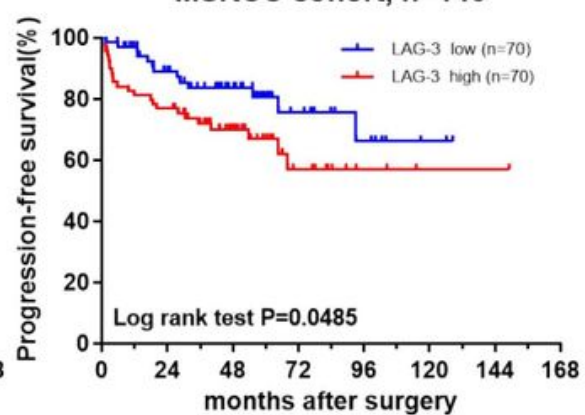

FUSCC cohort, $\mathrm{n}=175$

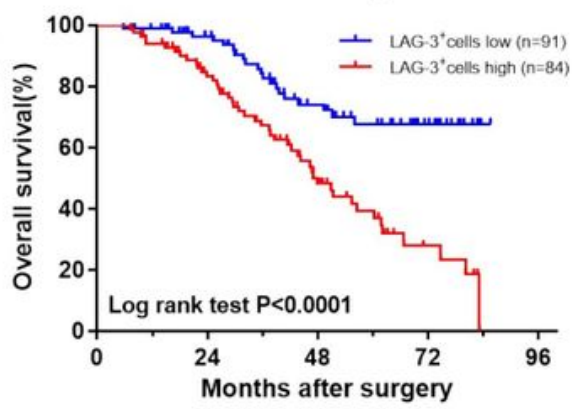

Intratumoral LAG-3+ T cells accumulate in castration-naïve prostate cancer and association with tumor progression.

\section{Figure 1}

Intratumoral LAG-3 + T cells accumulate in castration-naïve prostate cancer and correlate with tumor progression. (A) Representative immunohistochemistry images of LAG-3 + T cells infiltration in different tumor tissue compartments. (B) Kaplan-Meier analyses of PFS in TCGA cohort or MSKCC cohort 
according to LAG-3 mRNA expression. (C) Kaplan-Meier analyses of CRPC-FS and OS in FUSCC cohort according to intratumoral LAG-3 + T cells infiltration.
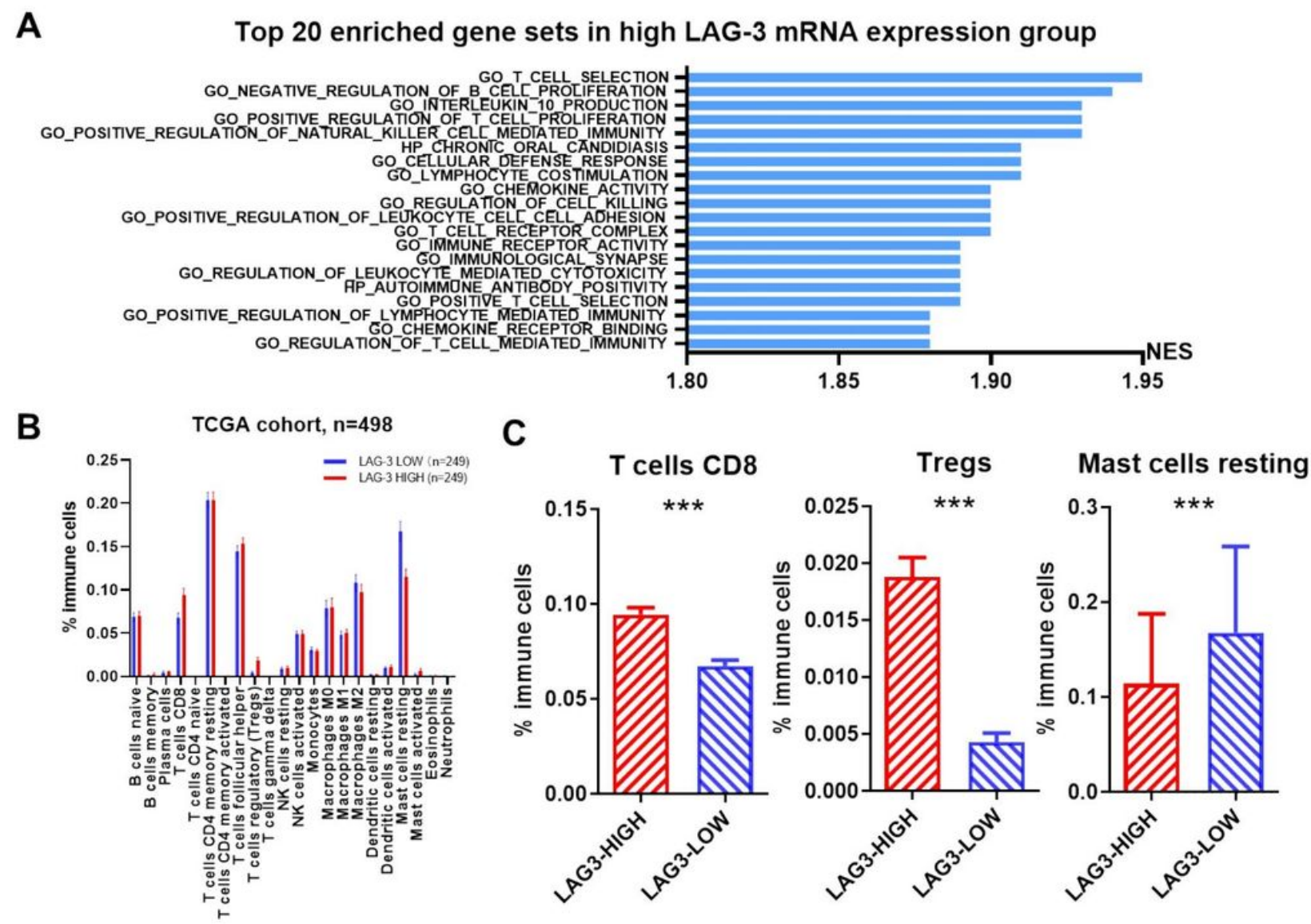

The differences of immune cell infiltration and biological process pathways according to LAG-3 mRNA expression in castration-naïve prostate cancer.

Figure 2

The differences of immune cell infiltration and biological process pathways according to LAG-3 mRNA expression in castration-naïve prostate cancer. (A) Top 20 enriched gene sets in high LAG-3 mRNA expression group derived from GSEA. Gene Otology Biological Process gene sets from MSigDB were used. 500 random sample permutations were performed. (B-C) Differences of immune cell infiltration according to LAG-3 mRNA expression in TCGA cohort(B) and specific groups with statistically differences(C). 

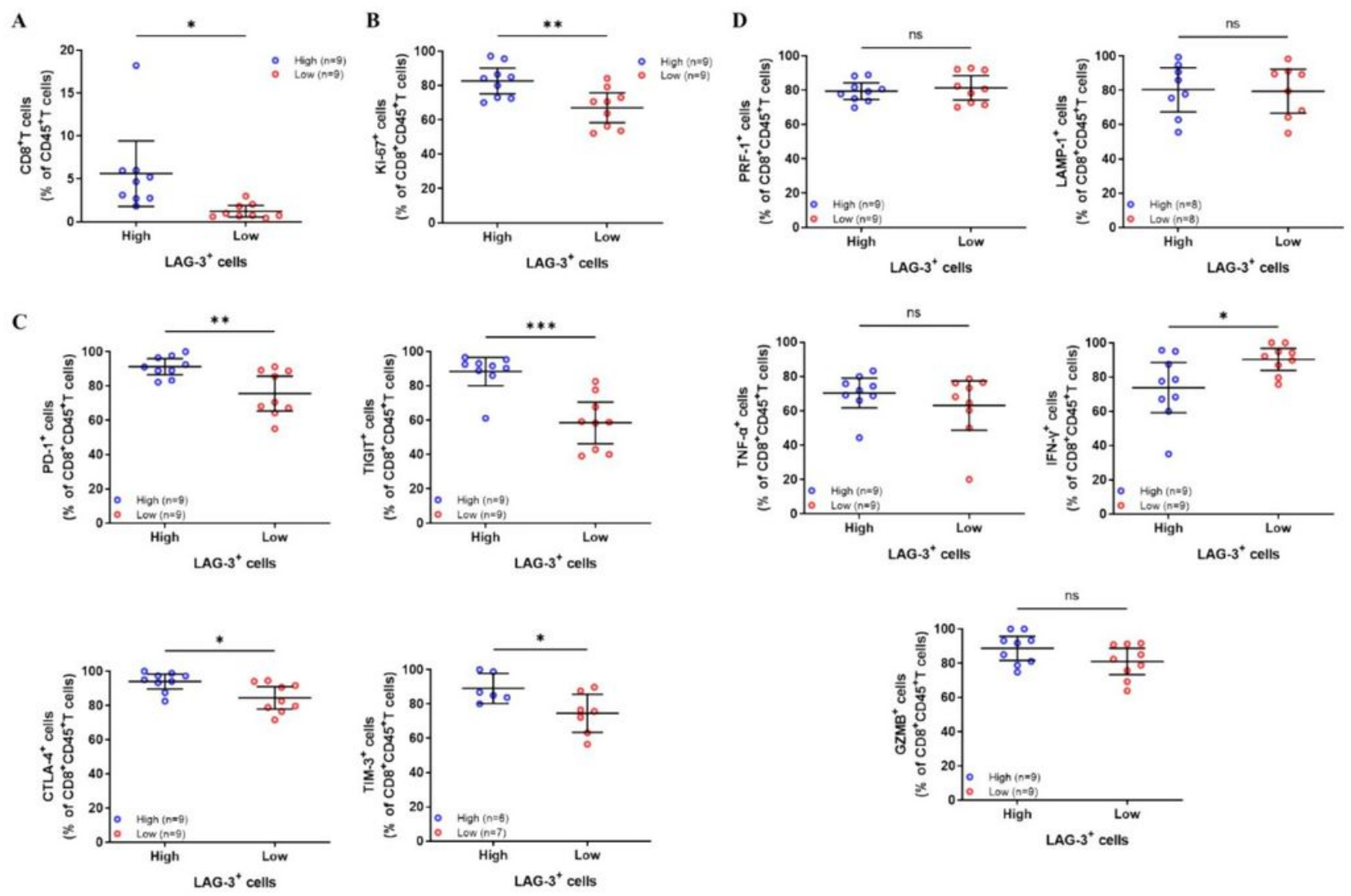

Intratumoral LAG-3 ${ }^{+} \mathrm{T}$ cell high infiltration impairs total $\mathrm{CD} 8^{+} \mathrm{T}$ cell immune function in patients with castration-naïve prostate cancer.

\section{Figure 3}

Intratumoral LAG-3 + T cell high infiltration impairs total CD8+ T cell immune function in patients with castration-naïve prostate cancer. (A) CD8+ T cell infiltration level in LAG-3 + T cell high/low subgroup. (B, C, D) Expression of immune proliferative marker, exhausted markers, activated markers, cytotoxic molecules and degranulation molecule for total CD8+T cells in LAG $\varangle 3+T$ cell high/low subgroup. All reported $p$ values were all two sided. IFN, interferon; PD-1, programmed cell-death protein 1.

\section{Supplementary Files}

This is a list of supplementary files associated with this preprint. Click to download.

- Tables.pdf

- SupplementTablesandFigures.docx 